\section{$\underset{\substack{\text { hommes } \\ \text { \& migrations }}}{ }$}

\section{Hommes \& migrations}

Revue française de référence sur les dynamiques

migratoires

\section{2 | 2018}

Exposer les migrations

\title{
Mai 68 et ses suites (re)vus par les enfants d'immigrés
}

\author{
Mogniss H. Abdallah
}

\section{OpenEdition}

\section{Journals}

Édition électronique

URL : https://journals.openedition.org/hommesmigrations/6849

DOI : 10.4000/hommesmigrations.6849

ISSN : 2262-3353

\section{Éditeur}

Musée national de l'histoire de l'immigration

\section{Édition imprimée}

Date de publication : 1 juillet 2018

Pagination : 190-196

ISBN : 978-2-919040-42-1

ISSN : $1142-852 X$

Référence électronique

Mogniss H. Abdallah, " Mai 68 et ses suites (re)vus par les enfants d'immigrés », Hommes \& migrations [En ligne], 1322 | 2018, mis en ligne le 01 juillet 2018, consulté le 21 janvier 2022. URL : http:// journals.openedition.org/hommesmigrations/6849; DOI : https://doi.org/10.4000/ hommesmigrations.6849 


\section{MAI 68 ET SES SUITES (RE)VUS PAR LES ENFANTS D'IMMIGRÉS}

Par MOGNISS H. ABDALLAH, journaliste, directeur de l'agence IM'média.

U n «Mai 68 des enfants d'immigrés»: la Marche pour l'égalité et contre le racisme de 1983 est parfois qualifiée comme tel, elle qui " a représenté une prise de parole nationale généralisée et qui a permis de socialiser des milliers de jeunes politiquement ${ }^{1}$ ». La formule peut paraître séduisante, pour autant elle risque d'occulter la question du regard des enfants d'immigrés sur Mai 68, comme témoins préadolescents ou pour certains déjà, comme acteurs. Ceux-là mêmes qui cherchaient à comprendre le pourquoi de l'effervescence ambiante d'alors, ou qui y firent leurs premières armes, et qui deviendront à des titres divers des cadres politiques ou culturels des années 1970 et 1980, ou du moins qui auront un rôle de trait d'union intergénérationnel et intercommunautaire, y compris donc pendant la Marche. Mais force est de constater par ailleurs que si, d'une commémoration à l'autre, quelques témoignages individuels épars ressurgissent au détour de récits de vie plus globaux, il y a une absence de réappropriation par l'immigration et ses héritiers du « moment 68 » comme une expérience collective fondatrice, à l'exception peut-être de la référence à certaines luttes de travailleurs immigrés durant les "années 68 " (RenaultBillancourt, Pennaroya, grève générale contre le racisme de 1973...). Une situation qui contraste, par exemple, avec le travail développé autour du massacre du 17 octobre 1961, ou de la Marche, afin de faire entrer ces événements dans l'Histoire. Pour comprendre ce reflux sélectif de mémoire, il est nécessaire de revenir sur les témoignages ou récits de vie existants, et de les recouper avec de nouveaux entretiens plus récents.

\section{Face à des parents réservés, une prise de conscience diffuse des enfants}

Dans son film documentaire Le Drôle de Mai (52 min, 2008), le réalisateur José Vieira revient, sous la forme d'une narration intimiste, sur ses vagues souvenirs d'enfance au bidonville de Massy - il avait dix ans au moment des "événements " de 68. Quarante ans plus tard, son père y confesse qu'il a été tenté, comme 10000 autres travailleurs portugais, de rentrer au pays «le temps que ça se tasse ». Mais sa mère a refusé. II faut dire que, pour le régime de Salazar et pour la Pide, police politique contrôlant étroitement l'immigration, la grève était considérée comme un "crime ", les communistes vus comme le "diable ", et évoquer le droit à la justice était de l'ordre du "blasphème". Étudiants et exilés portugais fuyant les guerres coloniales en Afrique contribueront en Mai 68 à la rupture avec cette " histoire subie ", entre autres grâce à la chanson engagée, comme le rappelle José Vieira - il connaît par cœur le répertoire de Luis Cilia - avec son film Le Printemps de l'exil (52 min, 2009). 


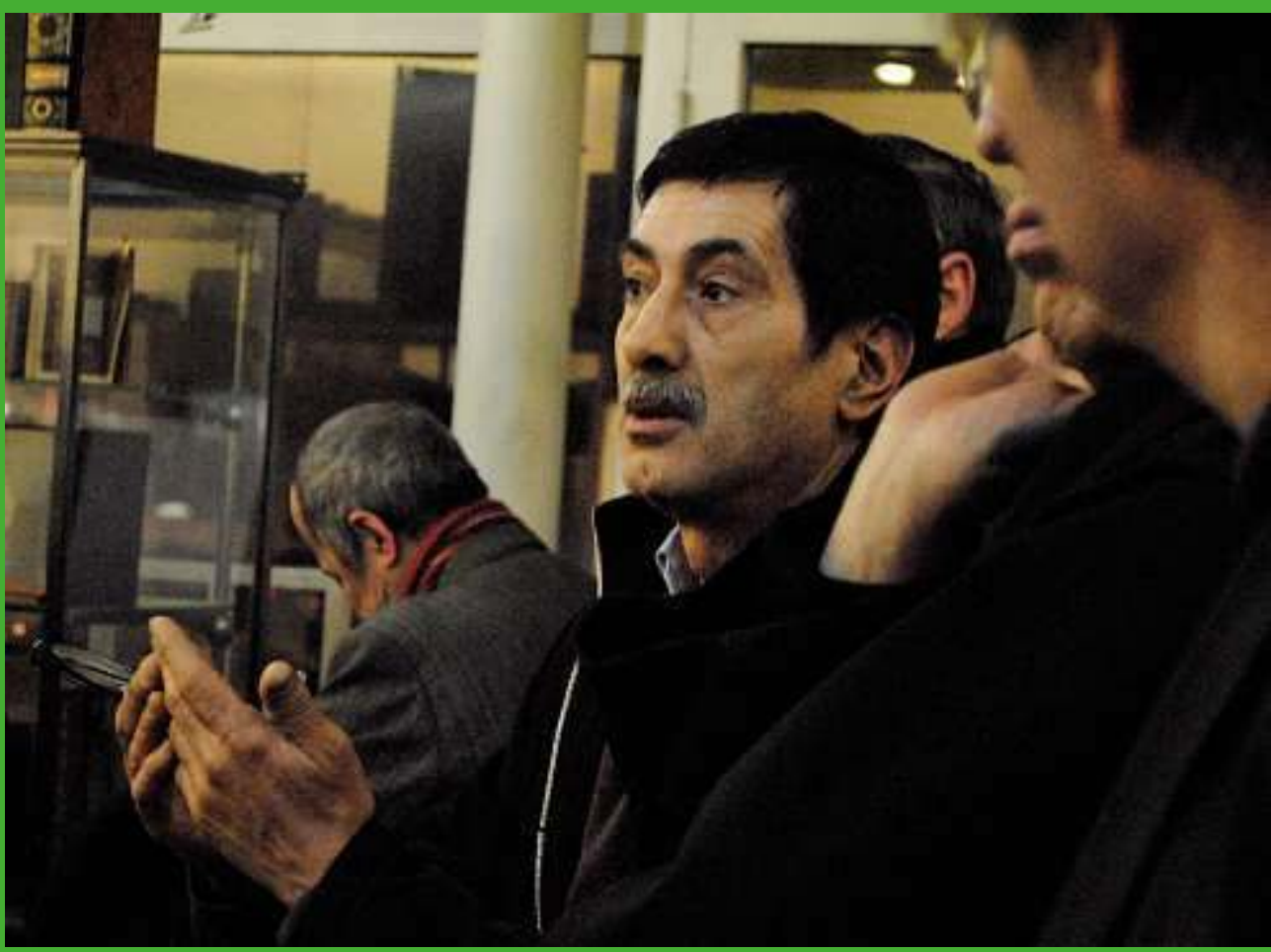

Cherif Cherfi. Photo Mognis Abdallah. () Mognis ABdALLAH, IM'MEDIA.

Brahim Benaïcha, alors âgé de 16 ans, habitait lui au bidonville rue des Pâquerettes à Nanterre, où les étudiants «enragés » de la nouvelle faculté limitrophe débarquaient pour prêcher la bonne parole. « Nos parents étaient choqués, raconte-t-il. Ils ne pouvaient pas apprendre la révolte, ils ne pouvaient pas se révolter... c'étaient des gens besogneux, moins ils faisaient de bruit, mieux ça valait... » Et luimême, n'a-t-il pas eu envie de se joindre à la révolte? "Non. On se serait fait casser la tête par nos parents! À l'époque, moi je disais que la dictature du prolétariat, ce n'était pas Georges Marchais, c'était le père, ouvrier spécialisé, qui disait "chut !"2.» Dans un livre autobiographique publié en 1992, Brahim Benaïcha précise que s'il a bien fréquenté les étudiants à la fac, il fut outré par leur violence, perçue comme un caprice d'enfants gâtés. "Ils ont tout et ils ne sont pas contents... Ils sont devenus fous ${ }^{3}$."

Autre jeune des bidonvilles de Nanterre, Mohamed Kenzi, 15 ans, témoigne, dans un récit publié en 1984, d'une ambiance similaire côté parental. « Les gens étaient effrayés. " "Les Français se battaient entre eux... ça ressemblait à la guerre. La communauté 
redevenait craintive, les blessures de la guerre d'Algérie n'étaient pas tout à fait cicatrisées 4 ! " Même ressentiment au bidonville Campagne Benausse à Marseille, d'après Hanifa Taguelmint, 7 ans. "Un jour, je vois débarquer deux jeunes (Isabelle et Richard) poursuivis par des flics. Ma mère les a planqués sous mon lit et me demande de me taire. " "Le soir, en traduisant à mon père les infos de la radio", il a dit: "S'ils s'en prennent à leurs jeunes, qu'est-ce qu'ils vont faire de nous 5 ? " "Mon père m'interdisait formellement de fréquenter ces individus » rajoute pour sa part Mohamed Kenzi, en parlant des étudiants. "Mais, comme chacun sait, l'interdit suscite un attrait tout particulier. C'était en cachette que je filais vers les lieux où ils se réunissaient. Leurs discours avaient ouvert une brèche dans mon esprit » et "fait mûrir un certain espoir de changement dans la tête des jeunes ${ }^{6} "$.

Persuadé de l'impossibilité d'une " conscientisation " au sein même de sa « communauté » du bidonville, et réfractaire au travail à l'usine, Mohamed Kenzi va militer quelque temps à titre individuel dans des groupes perçus comme "français " par ses pairs, fréquentera plus tard Vive la révolution, d'obédience " mao-spontex", ainsi que la " crèche sauvage" installée en 1970 pendant quelques mois à la fac des lettres de Nanterre. Son slogan : « Bébés françaisimmigrés, même biberon?. »

\section{Des fréquentations politiques ou culturelles multiples et paradoxales}

De fait, plusieurs dizaines de jeunes des bidonvilles ou des cités environnantes fréquentaient déjà la fac avant même Mai 68, pour accéder aux équipements collectifs sanitaires ou sportifs, pour manger au restau $U$, etc. Des fugueuses trouveront aussi refuge à la résidence universitaire auprès des étudiantes, alimentant de sordides qu'en dira-t-on au bidonville ou dans la presse à sensation.

"Avant, c'était notre terrain de jeu ", s'exclame Chérif Cherfi, en rappelant que la fac a été construite sur une aire d'aviation militaire à l'abandon. Arrivé d'Algérie dans les « baraques » en 1958, à l'âge de dix ans, il résidait alors à la cité Marcellin Berthelot, un bastion du PCF. II traversait souvent le campus parce que c'était un raccourci entre différents micro-quartiers de Nanterre où il avait ses amis. Ce faisant, il pratiquait un «droit à la ville ", peu ou prou conscient du «schéma d'alors: la ville européenne d'un côté, le douar de l'autre ${ }^{8}$ ".

Peu loquace sur sa participation directe aux événements de Mai 68, Chérif Cherfi révèle finalement, lors d'un entretien le 17 avril 2018 au théâtre Kalam à Colombes, qu'il a été en quelque sorte "manipulé " par ses contacts communistes à la Maison des jeunes de la Boule, qui l'ont embarqué dans la grande manif « syndicale » du 13 mai 1968 à Paris. Ses fréquentations politiques ou culturelles peuvent ainsi paraître paradoxales : il a commencé à militer dès 1967 au premier Comité Palestine de Nanterre aux côtés des jeunes de la cité de transit André Doucet, de jeunes ouvriers algériens et du sociologue Gilbert Mury, un « pro-chinois » connu pour sa virulence contre le "révisionnisme " du PCF. Et, en même temps, il garde de l'affection pour Raymond Barbet, le maire communiste, qui l'amenait parfois à l'école à bord de sa "dodoche". Il maintient des liens avec l'Amicale des Algériens en Europe, héritière de la Fédération de France du FLN, dont sa mère fut une membre active, malgré sa transformation en organe de contrôle de plus en plus décrié. Enfin, il restera en contact avec des militants chrétiens engagés dans la longue durée auprès des habitants des bidonvilles et des cités de transit, qui ont été parmi les premiers à s'occuper des enfants et à alerter sur leurs besoins spécifiques (école, activités extra-scolaires, logement...) ${ }^{9}$. 


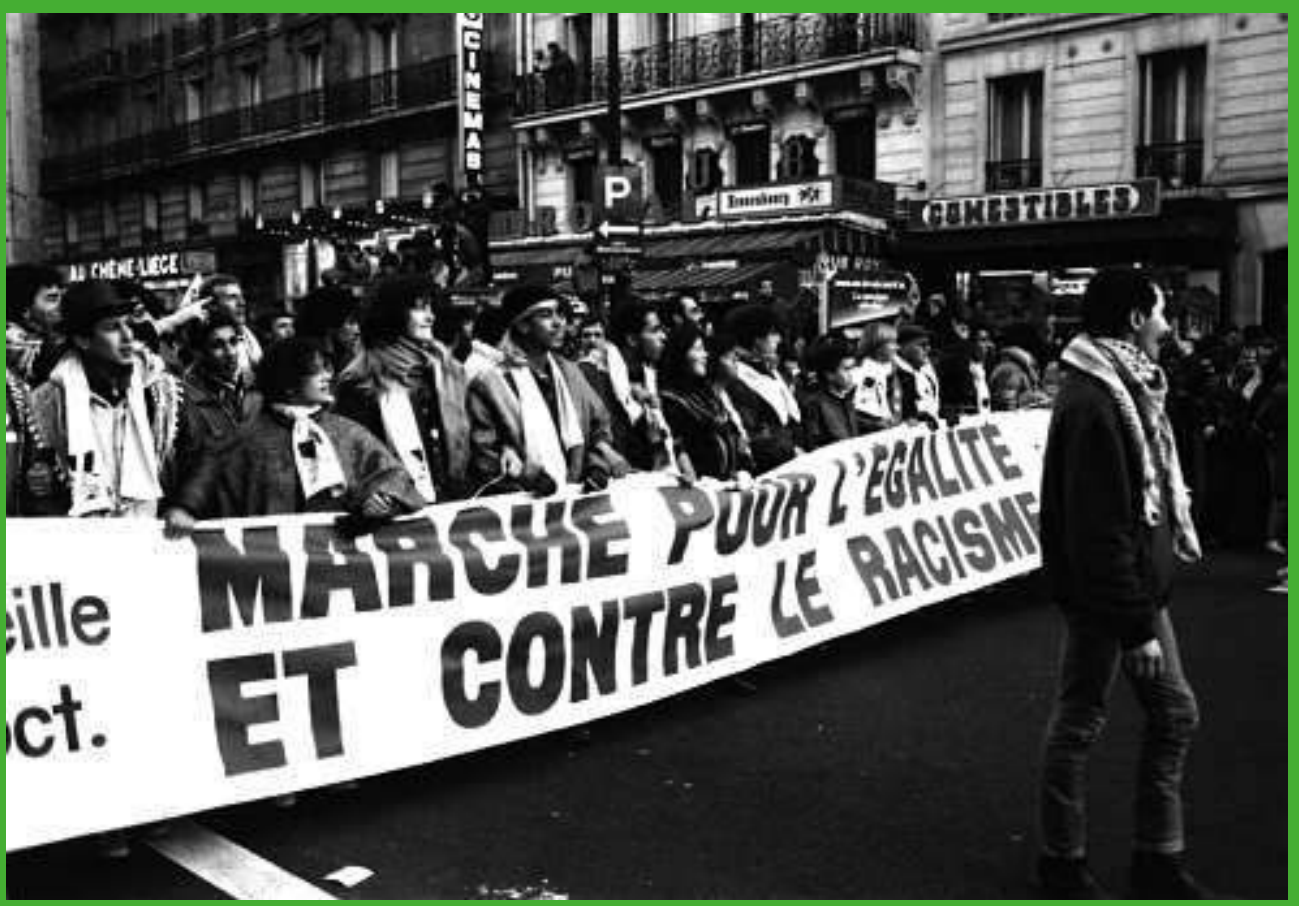

Farid Aichoune durant la Marche de 1983 à Paris.

Photo Mognis Abdallah. () Mognis Abdallah, IM'MEdiA.

S'il ne se définit pas explicitement comme " maoïste ", il se reconnaît bien dans la « révolution culturelle " chinoise et dans ses velléités de renverser les rapports de domination entre travail manuel et travail intellectuel. Lui-même a multiplié les petits boulots mais aspire à des activités culturelles, à travers notamment du théâtre et du cinéma militant. Entre-temps, tel un "robot ", il a collé des affiches et diffusé des tracts, vendu à la criée La Cause du peuple, journal à l'écrit-parlé qui, juge-t-il,formulait le mieux à l'époque ses idées de « résistance ».

\section{«Nous, la pègre!»}

Après la suspension des cours décidée le 2 mai 1968 par le recteur de la fac de Nanterre, certains jeunes des cités se retrouveront au Quartier latin, où ils croisent les "Katangais ", de "petits voyous de Montreuil10 " chargés de la sécurité dans la Sorbonne occupée, prêts à en découdre avec les militants du groupe d'extrême droite Occident. Mais c'est la violence "sauvage" de rue qui retiendra surtout l'attention dès le soir du 3 mai. Elle n'est pas le fait des seuls banlieusards. Des 


\section{REPÉRAGE}

étudiants, des badauds et même des anciens de 1936 s'en mêlent. Parmi les révoltés, des enfants d'immigrés aux allures de Gavroche qui vivaient dans les îlots d'habitat insalubre subsistant encore dans les ruelles adjacentes ou dans d'autres quartiers populaires de Paris. Farid Aïchoun, 16 ans, lycéen à Bergson, s'est politisé suite aux manifs contre la guerre au Vietnam mais aussi au contact de son père emprisonné du temps de son engagement au FLN. Le 10 mai, il s'est retrouvé à la « grande nuit des barricades " avec une bande de potes de son quartier «prolo » et multi-communautaire de Crimée (19e arrondissement). « La violence ne nous était pas étrangère. Elle était d'abord sociale ", se remémore-t-il, en citant Brassens : "Quand il s'agit de rosser les cognes, tout le monde se réconcilie"... On avait une revanche à prendre sur les flics, leurs brimades, leurs contrôles, le massacre de Charonne et du 17 octobre $1961^{11}$. "

Le 25 mai 1968 Christian Fouchet, ministre de I'Intérieur, tonne : "Je demande à Paris de "vomir" cette pègre qui la déshonore. Cette pègre, qui sort des bas-fonds de Paris et qui est véritablement enragée, dissimulée derrière les étudiants, se bat avec une folie meurtrière. C'est le rôle du gouvernement de mettre fin le plus vite possible à l'action de cette pègre ". L'Humanité dimanche du 26 mai 1968 renchérit: "On trouve une racaille des plus douteuse... dont la présence contamine ceux qui l'acceptent et, plus encore, ceux qui la sollicitent. " Un nouveau tournant s'opère : celui de la racialisation des « blousons noirs » et de la stigmatisation des « délinquants étrangers », a fortiori des jeunes. Par extension, les manifestants dénoncent des "ratonnades» et un racisme policier "antijeunes". Les scènes de répression révulsent les témoins, à Paris mais aussi à Lyon, où, le 24 mai, plus de 150 jeunes ou immigrés sont arrêtés après des descentes policières dans les meublés du quartier de la Guillotière. Une trentaine sera expulsée du territoire ${ }^{12}$. Les expulsions, de plus en plus nombreuses, ciblent indistinctement étudiants, travailleurs, chômeurs, adolescents, toutes nationalités étrangères confondues ${ }^{13}$. Souvent motivées pour " non respect de la stricte neutralité politique ", elles symbolisent - au-delà du cas célèbre de Daniel Cohn-Bendit - le déni de liberté d'expression et d'association pour les étrangers.

"La chienlit, c'est lui » proclame, silhouette du général De Gaulle à l'appui, une des célèbres affiches des Beaux-arts. Par retournement du stigmate, un tract intitulé « Nous, la Pègre » circule en réponse à Fouchet, et Dominique Grange chante "La pègre on en est / La chienlit aussi / Des éléments parfait'ment incontrôlés / Des indésirables... " Cependant, une partie du mouvement étudiant s'interroge sur la place des jeunes immigrés: "À Nanterre, écrit un membre du Mouvement du 22 mars, il se trouve que le service d'ordre de la faculté est assuré par des Nord-Africains qui sont utilisés comme des chiens de garde et qui n'ont absolument pas de contact avec le mouvement proprement dit... Je ne suis pas contre le fait que ces Nord-Africains fassent partie du service d'ordre, mais il faut qu'ils comprennent pourquoi ils le font ${ }^{14} \ldots$..

Mi-juin, à la Sorbonne, les membres du «S.O bis » des "Katangais ", soupçonnés d'être infiltrés et accusés de multiples méfaits au sein même de l'université occupée, sont évacués manu militari par l'extrême gauche ${ }^{15}$. Cet épisode va marquer durablement les esprits, augurant de tensions ultérieures entre les jeunes et certains groupes politiques. Les maos vont se montrer plus indulgents à l'égard de ceux du « lumpen-prolétariat", "chargés de toutes les basses tâches". "Mais dès lors qu'il s'agit de s'organiser politiquement, dans les Comités d'action ou dans les groupes politiques, le lumpen s'absente... Ces éléments ne sont pas des "militants"16..." 


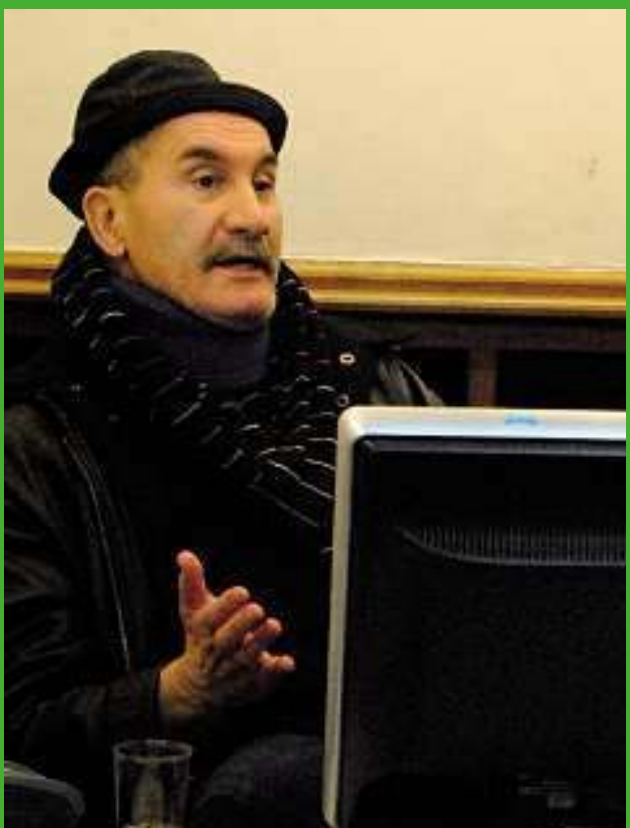

Farid Aichoune. Photo Mognis Abdallah (c) MogNIS ABdALLAH, IM'MEDIA.

\section{S’organiser...}

La question de l'organisation, dans le cadre d'une "lutte prolongée » se pose, en effet, comme le constate l'écrivain algérien Kateb Yacine : "Je me suis demandés'il y avait une force qui les organisait (les immigrés algériens)... Ils étaient là, mais il n'y avait pas de possibilité d'orienter leur action faute d'organisation propre auximmigrés, faute de liaison avec les jeunes et avec les organisations pour s'inscrire dans le grand mouvement des luttes françaises ${ }^{17}$. » Mais elle se pose à tous les protagonistes de Mai 68, pas seulement aux immigrés ou au "lumpen". Or le mouvement d'ensemble, telle une nébuleuse éphémère, verra nombre de ses structures ad hoc disparaître dès la reprise en main de la situation par le pouvoir gaulliste. En outre, la dissolution par décret, le 12 juin 1968, de la plupart des groupes "gauchistes » va précipiter beaucoup de militants dans une clandestinité plus ou moins grande, les poussant à prendre des pseudos ou à parler sous le couvert de l'anonymat. Et les affrontements se durciront.

Mohamed Kenzi amorce dès lors sa sortie du "gauchisme", mais sera rattrapé par son passé et expulsé. Chérif Cherfi, alias "Charlie », et Farid Aïchoune s'engagent pour leur part activement au Mouvement des travailleurs (MTA), apparu en juin 1972 dans la foulée des Comités Palestine. Le MTA, parfois considéré comme la « branche immigrée » des maoïstes de la Gauche prolétarienne (GP), est un mouvement d'inspiration marxiste et pan-arabe implanté dans plusieurs régions, composé en majorité d'étudiants ou d'ouvriers tunisiens et marocains. Dans son « noyau » dirigeant, Saïd Bouziri et Méjid Daboussi, alias "Ammar ", ont tous deux été omniprésents dans le mouvement étudiant en Mai 68, l'un à Lyon, l'autre à Paris. Partisans de l'auto-organisation des immigrés de différentes nationalités, ils n'ont pas oublié que les revendications dites "spécifiques » (dont la suppression des contrats provisoires, la promotion sociale avec les mêmes critères que l'ensemble du personnel, l'égalité des droits syndicaux) ont été sacrifiées sur l'autel des accords de Grenelle du 27 mai 196818. Pour autant, ils prônent aussi l'unité avec les travailleurs français (en particulier au sein de l'Union nationale des comités de lutte d'atelier, UNCLA) et avec les "démocrates". Ils manifestent également une prédisposition à la médiation avec la direction de syndicats ou de partis politiques.

Il s'agit désormais d'en finir avec le style d'intervention de "groupe d'agitateurs » et de se structurer efficacement en organisation de masse à même de donner une cohérence politique d'ensemble aux multiples " fronts de lutte " par 


\section{REPÉRAGE}

trop éparpillés : mobilisations contre les crimes racistes, contre l'expulsion de plusieurs militants dont Saïd et Fawzia Bouziri, occupations d'usines pour des conditions de travail et de salaires décentes, actions des mal-logés, luttes des sanspapiers, grève générale contre le racisme de Marseille à Paris... Le MTA réussit parfois à recouper ces luttes. Ce sera, par exemple, le cas avec la candidature de « Djellali Kamal ", un sans-papiers exgréviste de la faim, à l'élection présidentielle de 1974. Cette initiative symbolique réactivera de façon spectaculaire les revendications de « liberté d'expression et d'association pour les immigrés » et d' "égalité des droits conquis par l'ensemble des travailleurs de France ${ }^{19}$ ".

\section{...pour vivre « ici et maintenant »}

Chérif Cherfi et Farid Aïchoune participent, chacun à sa manière, à plusieurs de ces luttes. La présence à Barbès d'intellectuels de renom comme Jean-Paul Sartre, Michel Foucault ou Claude Mauriac, aux côtés des luttes immigrées, ainsi que celle d'étudiants arabes politisés, les captive. "Mais le pouvoir ne doit pas rester entre les mains des intellectuels ", dit-on aussi au MTA, sourcilleux en matière d'autonomie, tant dans l'action que dans le domaine de la culture.

Aussi, l'un et l'autre reprennent-ils des études afin de compléter leur formation et voyagent aux grés de leurs idéaux tiers-mondistes. Installé à la résidence universitaire de Nanterre, Chérif Cherfi s'inscrit en auditeur libre à la fac, puis en licence de cinéma à l'université de Vincennes, accessible aux non-diplômés. Son intérêt pour le cinéma militant va lui permettre de développer son réseau d'interconnaissances et de s'ouvrir à de nouveaux combats, comme celui du Mouvement pour la liberté d'avortement et de contraception (MLAC), avec lequel il assure la projection de films interdits. Côté théâtre, il donne un coup de main à plusieurs troupes dont Al Assifa, qui émane du MTA, La Kahina ou Weekend à Nanterre. Farid Aïchoune, lui, entreprend au mitan des années 1970 des études d'histoire-géo qui affermiront son goût pour l'enquête et le journalisme. Fort en gueule, il sera une des figures marquantes de l'hebdomadaire Sans Frontière, lancé au printemps 1979 par une partie des anciens du MTA, rejoints par une kyrielle d'apprentis-journalistes ou de contributeurs occasionnels qui trouvent là un exutoire à leur besoin de s'exprimer, non sans conflits internes.

L'ébullition culturelle des années 1970 favorise ainsi l'irruption sur la scène publique de la vie familiale, des aspirations des femmes et des jeunes immigrés, des enjeux pas ou peu abordés jusqu'alors, y compris par les militants immigrés eux-mêmes. La filiation avec l'esprit de Mai 68 transparaît en filigrane : l'aspiration à « vivre ici et maintenant " l'emporte désormais sur le sempiternel mythe du retour. Et les immigrés ne sont plus réductibles à leur seule force de travail.

Les parcours individuels que nous avons pu suivre ici, au-delà de leurs singularités propres, dénotent la prédisposition de ces militants, ou ex-militants encore actifs par ailleurs, à devenir des passeurs ou des médiateurs entre les générations des parents et celles des jeunes, des années 1980 à aujourd'hui. Un des moments emblématiques de ce rôle de trait d'union a sans doute été la manifestation commune entre des licenciés de Talbot-Poissy et des Marcheurs pour l'égalité, début 1984 à Paris. Ou encore les différentes campagnes pour le droit de vote de tous les résidents étrangers qui signifierait pour les héritiers de l'immigration une reconnaissance symbolique de leurs parents et de leur droit de cité dans la société française. Et qui rappelle que, pour eux, la lutte sans cesse recommencée contre les discriminations entre Français ne saurait être dissociée de l'égalité des droits entre Français et immigrés. 